\title{
Existence and Characterization of Solutions of Nonlinear Volterra-Stieltjes Integral Equations in Two Variables
}

\author{
Mohamed Abdalla Darwish ${ }^{1}$ and Józef Banaś ${ }^{2}$ \\ ${ }^{1}$ Mathematics Department, Science Faculty for Girls, King Abdulaziz University, Jeddah 21589, Saudi Arabia \\ ${ }^{2}$ Department of Mathematics, Rzeszów University of Technology, Aleja Powstańców Warszawy 8, 35-959 Rzeszów, Poland
}

Correspondence should be addressed to Józef Banaś; jbanas@prz.edu.pl

Received 7 February 2014; Accepted 29 April 2014; Published 18 May 2014

Academic Editor: Cemil Tunç

Copyright (C) 2014 M. A. Darwish and J. Banaś. This is an open access article distributed under the Creative Commons Attribution License, which permits unrestricted use, distribution, and reproduction in any medium, provided the original work is properly cited.

The paper is devoted mainly to the study of the existence of solutions depending on two variables of a nonlinear integral equation of Volterra-Stieltjes type. The basic tool used in investigations is the technique of measures of noncompactness and Darbo's fixed point theorem. The results obtained in the paper are applicable, in a particular case, to the nonlinear partial integral equations of fractional orders.

\section{Introduction}

The theory of differential and integral equations of fractional order creates nowadays a large subject of mathematics which found in the last three decades numerous applications in physics, mechanics, engineering, bioengineering, viscoelasticity, electrochemistry, control theory, porous media, and other fields connected with real world problems [1-5]. Let us mention that recently there have appeared a few important and expository monographs covering both the theory and applications of differential and integral equations of fractional order (cf. $[1,4,6-8])$.

It turns out that a lot of results of the theory of differential and integral equations of fractional order can be considered from a unified point of view with help of the theory of the so-called Volterra-Stieltjes integral equations (cf. $[9,10])$. The approach applied in those papers allows us not only to consider the mentioned theories of differential and integral equations of fractional order from one point of view but also to obtain deeper results with help of less complicated tools of nonlinear analysis.

Such an approach can be also applied to investigations associated with the theory of differential and integral equations of fractional orders in two variables. That subject of nonlinear analysis was recently studied in a few papers [11-16]. It seems that results obtained in those papers are not sufficiently general. The tools and methods associated with the theory of nonlinear Volterra-Stieltjes integral equations which will be applied in this paper are more convenient and allow us to obtain more applicable results. Indeed, further on we obtain, as particular cases, the existence theorems concerning both nonlinear integral equations of fractional orders, nonlinear integral equations of Volterra-Chandrasekhar type, and nonlinear equations of mixed type. Moreover, we indicate possible generalizations of our results to the situation of nonlinear Volterra-Stieltjes integral equations in $n$ variables. Additionally, we indicate also a few open problems appearing in our theory.

\section{Notation, Definitions, and Auxiliary Results}

This section is devoted to provide the notation, definitions, and other auxiliary facts which will be needed in our further study.

At the beginning let us assume that $f$ is a real function defined on the interval $[a, b]$. Then the symbol $\bigvee_{a}^{b} f$ will denote the variation of the function $f$ on the interval $[a, b]$. In the case when $\bigvee_{a}^{b} f<\infty$ we say that $f$ is of bounded variation on $[a, b]$. If we have a function $u(t, x)=u:[a, b] \times[c, d] \rightarrow$ $\mathbb{R}$, then we denote by $\bigvee_{t=p}^{q} u(t, x)$ the variation of the function 
$t \rightarrow u(t, x)$ on the interval $[p, q] \subset[a, b]$. Similarly we define the quantity $\bigvee_{x=p}^{q} u(t, x)$.

For the properties of functions of bounded variation we refer to [17].

If $f$ and $\varphi$ are two real functions defined on the interval $[a, b]$, then under some additional conditions $[17,18]$ we can define the Stieltjes integral (in the Riemann-Stieltjes sense)

$$
\int_{a}^{b} f(t) d \varphi(t)
$$

of the function $f$ with respect to the function $\varphi$. In this case we say that $f$ is Stieltjes integrable on the interval $[a, b]$ with respect to $\varphi$.

It is worthwhile mentioning that several conditions ensuring Stieltjes integrability may be found in [17]. One of the most frequently used requires $f$ to be continuous and $\varphi$ to be of bounded variation on $[a, b]$.

In the sequel we will utilize a few properties of the Stieltjes integral contained in the below quoted lemmas (cf. [17]).

Lemma 1. If $f$ is Stieltjes integrable on the interval $[a, b]$ with respect to a function $\varphi$ of bounded variation, then

$$
\left|\int_{a}^{b} f(t) d \varphi(t)\right| \leq \int_{a}^{b}|f(t)| d\left(\bigvee_{a}^{t} \varphi\right)
$$

Lemma 2. Let $f_{1}, f_{2}$ be Stieltjes integrable functions on the interval $[a, b]$ with respect to a nondecreasing function $\varphi$ such that $f_{1}(t) \leq f_{2}(t)$ for $t \in[a, b]$. Then

$$
\int_{a}^{b} f_{1}(t) d \varphi(t) \leq \int_{a}^{b} f_{2}(t) d \varphi(t) .
$$

In what follows we will also consider Stieltjes integrals having the form

$$
\int_{a}^{b} f(t) d_{s} g(t, s),
$$

where $g:[a, b] \times[a, b] \rightarrow \mathbb{R}$ and the symbol $d_{\text {s }}$ indicates the integration with respect to the variable $s$. The details concerning the integral of this type will be given later.

Even more, in our considerations we will use the double Stieltjes integrals of the form

$$
\int_{c}^{d} \int_{c}^{d} f(t, x) d_{y} g_{2}(x, y) d_{s} g_{1}(t, s),
$$

where $g_{i}:[a, b] \times[c, d] \rightarrow \mathbb{R}(i=1,2)$. Obviously, the double Stieltjes integral (5) is understood as the following double iterated Stieltjes integral:

$$
\int_{c}^{d}\left(\int_{c}^{d} f(t, x) d_{y} g_{2}(x, y)\right) d_{s} g_{1}(t, s) .
$$

Now, let us assume that $u=u(t, x)$ is a real function defined on the Cartesian product $[a, b] \times[c, d]$. Denote by $\omega(u, \varepsilon)$ the modulus of continuity of the function $u$; that is,

$$
\begin{gathered}
\omega(u, \varepsilon)=\sup \{|u(t, x)-u(s, y)|: t, s \in[a, b], x, y \in[c, d], \\
|t-s| \leq \varepsilon,|x-y| \leq \varepsilon\} .
\end{gathered}
$$

Obviously, we can also consider the modulus of continuity of the function $u(t, x)$ with respect to each variable separately. For example,

$$
\begin{gathered}
\omega(u(t, \cdot), \varepsilon)=\sup \{|u(t, x)-u(t, y)|: x, y \in[c, d], \\
|x-y| \leq \varepsilon\},
\end{gathered}
$$

where $t$ is a fixed number in the interval $[a, b]$.

Further on, in order to simplify our investigations, we will always assume that $[a, b]=[c, d]=[0,1]$ and we will denote by $I$ the unit interval $[0,1]$; that is, $I=[0,1]$.

Now, we recall some facts concerning measures of noncompactness, which will be applied in the sequel. To this end assume that $E$ is an infinite dimensional Banach space with the norm $\|\cdot\|$ and zero element $\theta$. Denote by $B(x, r)$ the closed ball centered at $x$ and radius $r$. The symbol $B_{r}$ stands for the ball $B(\theta, r)$.

Next, for a given nonempty bounded subset $X$ of $E$, we denote by $\chi(X)$ the so-called Hausdorff measure of noncompactness of the set $X$ [19]. This quantity is defined by the formula

$$
\chi(X)=\inf \{\varepsilon>0: X \text { has a finite } \varepsilon-\text { net in } E\} .
$$

Let us mention that the function $\chi$ has several useful properties and is often applied in nonlinear analysis, operator theory, and the theories of differential and integral equations $[19,20]$.

Notice that the concept of a measure of noncompactness may be defined in a more general way [19, 21], but for our purposes the Hausdorff measure of noncompactness defined by (9) will be thoroughly sufficient.

In fact, in our further considerations, we will work in the space $C\left(I^{2}\right)$ consisting of all functions $u=u(t, x)$ defined and continuous on the Cartesian product $I^{2}=I \times I$ with real values. The space $C=C\left(I^{2}\right)$ will be furnished with the standard maximum norm

$$
\|u\|_{C}=\max \left\{|u(t, x)|:(t, x) \in I^{2}\right\} .
$$

It can be shown [19] that if $U$ is a nonempty and bounded subset of $C\left(I^{2}\right)$, then the Hausdorff measure of noncompactness of $U$ can be expressed by the following formula:

$$
\chi(U)=\frac{1}{2} \omega_{0}(U)
$$

where

$$
\omega_{0}(U)=\lim _{\varepsilon \rightarrow 0} \omega(U, \varepsilon)
$$

The symbol $\omega(U, \varepsilon)$ used above denotes the modulus of continuity of the set $U$ and is defined as follows:

$$
\omega(U, \varepsilon)=\sup \{\omega(u, \varepsilon): u \in U\}
$$

while $\omega(u, \varepsilon)$ stands for the modulus of continuity of the function $u$ defined by (7).

Now we recall a fixed point theorem of Darbo type which will be utilized in our investigations (cf. [19]). 
Theorem 3. Let $\Omega$ be a nonempty, bounded, closed, and convex subset of the Banach space $E$ and let $Q: \Omega \rightarrow \Omega$ be a continuous operator such that there exists a constant $k \in[0,1)$ for which $\chi(Q U) \leq k \chi(U)$ provided $U$ is an arbitrary nonempty subset of $\Omega$. Then $Q$ has at least one fixed point in the set $\Omega$.

Next we recall a few facts concerning the so-called superposition operator [19]. To this end assume that $D=I^{2}$, where $I=[0,1]$. Let $f: D \times \mathbb{R} \rightarrow \mathbb{R}$ be a given function. Then, to every function $u$ acting from $I^{2}$ into $\mathbb{R}$ we may assign the function $F u$ defined by the formula

$$
(F u)(t, x)=f(t, x, u(t, x))
$$

for $(t, x) \in I^{2}$. The operator $F$ defined in such a way is called the superposition operator generated by the function $f=f(t, x, u)$.

The properties of the superposition operator may be found in [22]. For our further purposes we will only need the below quoted result concerning the behaviour of the superposition operator $F$ in the space $C\left(I^{2}\right)$.

Lemma 4. The superposition operator $F$ generated by the function $f: I^{2} \times \mathbb{R} \rightarrow \mathbb{R}$ transforms the space $C\left(I^{2}\right)$ into itself and is continuous if and only if the function $f$ is continuous on the set $I^{2} \times \mathbb{R}$.

Remark 5. Let us notice that in our considerations concerning the superposition operator $F$ generated by the function $f$ we may replace the set $D=I^{2}$ by an arbitrary Cartesian product $D=I^{2}$ with $I=[a, b]$ or even by $D=[a, b] \times[c, d]$ (cf. [22] for further possible generalizations).

Finally, we recall some fundamental facts associated with fractional calculus (cf. $[7,8,23])$. To this end denote by $L^{1}(a, b)$ the space of all real functions defined and Lebesgue integrable on the interval $(a, b)$. The space $L^{1}(a, b)$ is equipped with the standard norm. Further, fix a number $\alpha>0$ and take an arbitrary function $u \in L^{1}(a, b)$. The Riemann-Liouville fractional integral of order $\alpha$ of the function $u=u(t)$ is defined by the formula

$$
I^{-\alpha} u(t)=\frac{1}{\Gamma(\alpha)} \int_{a}^{t} \frac{u(s)}{(t-s)^{1-\alpha}} d s, \quad t \in(a, b),
$$

where $\Gamma(\alpha)$ denotes the gamma function.

It may be shown that the fractional integral operator $I^{-\alpha}$ transforms the space $L^{1}(a, b)$ into itself and has some additional properties $[7,8,23]$.

\section{Main Result}

Investigations of this paper are connected mainly with the solvability of the following nonlinear quadratic integral equation of Volterra-Stieltjes type having the form

$$
\begin{aligned}
u(t, x)= & h(t, x)+f(t, x, u(t, x)) \\
& \times \int_{0}^{t} \int_{0}^{x} v(t, s, x, y, u(s, y)) d_{y} g_{2}(x, y) d_{s} g_{1}(t, s),
\end{aligned}
$$

for $(t, x) \in I^{2}$, where $I=[0,1]$.

Let us recall that details concerning the notation used in (16) were presented in the previous section.

In order to formulate the assumptions under which (16) will be investigated, let us denote by $\Delta_{i}(i=1,2)$ the following triangles:

$$
\begin{aligned}
& \Delta_{1}=\{(t, s): 0 \leq s \leq t \leq 1\}, \\
& \Delta_{2}=\{(x, y): 0 \leq y \leq x \leq 1\} .
\end{aligned}
$$

We will study (16) assuming the following hypotheses:

(i) $h \in C\left(I^{2}\right)$;

(ii) the function $f(t, x, u)=f: I^{2} \times \mathbb{R} \rightarrow \mathbb{R}$ is continuous and satisfies the Lipschitz condition with respect to the variable $\mathcal{u}$; that is, there exists a constant $k>0$ such that

$$
|f(t, x, u)-f(t, x, w)| \leq k|u-w|
$$

for all $t, x \in I$ and $u, w \in \mathbb{R}$;

(iii) the function $g_{i}(w, z)=g_{i}: \Delta_{i} \rightarrow \mathbb{R}$ is continuous on the triangle $\Delta_{i}$ for $i=1,2$;

(iv) the function $z \rightarrow g_{i}(w, z)$ is of bounded variation on the interval $[0, w]$ for each fixed $w \in I(i=1,2)$;

(v) for any $\varepsilon>0$ there exists $\delta>0$ such that, for all $w_{1}, w_{2} \in I, w_{1}<w_{2}$, and $w_{2}-w_{1} \leq \delta$, the following inequality is satisfied:

$$
\bigvee_{z=0}^{w_{1}}\left[g_{i}\left(w_{2}, z\right)-g_{i}\left(w_{1}, z\right)\right] \leq \varepsilon
$$

for $i=1,2$;

(vi) $g_{i}(w, 0)=0$ for each $w \in I(i=1,2)$;

(vii) $v: \Delta_{1} \times \Delta_{2} \times \mathbb{R} \rightarrow \mathbb{R}$ is a continuous function such that

$$
|v(t, s, x, y, u)| \leq \phi(|u|)
$$

for all $(t, s) \in \Delta_{1},(x, y) \in \Delta_{2}$ and for each $u \in \mathbb{R}$, where $\phi: \mathbb{R}_{+} \rightarrow \mathbb{R}_{+}$is a nondecreasing function.

Before formulating further assumptions concerning (16) we provide a few lemmas proved in [9] which will be utilized in our investigations.

Lemma 6. The function

$$
p \longrightarrow \bigvee_{z=0}^{p} g_{i}(w, z)
$$

is continuous on the interval $[0, w]$ for any $w \in I(i=1,2)$. 
Lemma 7. Let assumptions (iii)-(v) be satisfied. Then, for arbitrarily fixed number $w_{2} \in I\left(w_{2}>0\right)$ and for any $\varepsilon>0$ there exists $\delta>0$ such that if $w_{1} \in I, w_{1}<w_{2}$, and $w_{2}-w_{1} \leq \delta$, then

$$
\bigvee_{z=w_{1}}^{w_{2}} g_{i}\left(w_{2}, z\right) \leq \varepsilon
$$

$(i=1,2)$.

Lemma 8. Under assumptions (iii)-(v) the function

$$
w \longrightarrow \bigvee_{z=0}^{w} g_{i}(w, z)
$$

is continuous on the interval $I(i=1,2)$.

As an immediate consequence of the above lemma we derive the following corollary.

Corollary 9. There exists a finite positive constant $K_{i}$ such that

$$
K_{i}=\sup \left\{\bigvee_{z=0}^{w} g_{i}(w, z): w \in I\right\}
$$

$(i=1,2)$.

In what follows let us denote by $F_{1}$ the constant defined by the formula

$$
F_{1}=\max \{|f(t, x, 0)|: t, x \in I\} .
$$

Obviously, in view of assumption (ii) we have that $F_{1}<\infty$. on.

Now, we can formulate the last assumption used further

(viii) There exists a positive solution $r_{0}$ of the inequality

$$
\|h\|_{C}+\left(k r+F_{1}\right) K_{1} K_{2} \phi(r) \leq r
$$

such that

$$
k K_{1} K_{2} \phi\left(r_{0}\right)<1 \text {. }
$$

Our main result is contained in the following theorem.

Theorem 10. Under assumptions (i)-(viii) there exists at least one solution $u=u(t, x)$ of $(16)$ in the space $C=C\left(I^{2}\right)$.

Proof. We start with the following notation:

$$
\begin{aligned}
M_{i}(\varepsilon)=\sup \left\{\bigvee_{z=0}^{w_{1}}\left[g_{i}\left(w_{2}, z\right)-g_{i}\left(w_{1}, z\right)\right]:\right. \\
\left.w_{1}, w_{2} \in I, w_{1}<w_{2}, w_{2}-w_{1} \leq \varepsilon\right\}
\end{aligned}
$$

for $i=1,2$. Notice that in view of assumption (v) we infer that $M_{i}(\varepsilon) \rightarrow 0$ as $\varepsilon \rightarrow 0$ for $i=1,2$.
Moreover, for further purposes let us define the function $N_{i}=N_{i}(\varepsilon)(i=1,2)$ by putting

$$
\begin{gathered}
N_{i}(\varepsilon)=\sup \left\{\bigvee_{z=w_{1}}^{w_{2}} g_{i}\left(w_{2}, z\right): w_{1}, w_{2} \in I, w_{1}<w_{2},\right. \\
\left.w_{2}-w_{1} \leq \varepsilon\right\} .
\end{gathered}
$$

Observe that in virtue of Lemma 7 we have that $N_{i}(\varepsilon) \rightarrow 0$ as $\varepsilon \rightarrow 0(i=1,2)$.

Further, for a fixed function $u \in C$ and $t, x \in I$, let us denote

$$
(F u)(t, x)=f(t, x, u(t, x)),
$$

$(V u)(t, x)$

$$
\begin{aligned}
= & \int_{0}^{t} \int_{0}^{x} v(t, s, x, y, u(s, y)) d_{y} g_{2}(x, y) d_{s} g_{1}(t, s), \\
& (\mathrm{Qu})(t, x)=h(t, x)+(F u)(t, x)(V u)(t, x) .
\end{aligned}
$$

Next, fix arbitrarily $\varepsilon>0$ and choose $t_{1}, t_{2}, x_{1}, x_{2} \in I$ such that $t_{1} \leq t_{2}, x_{1} \leq x_{2}, t_{2}-t_{1} \leq \varepsilon, x_{2}-x_{1} \leq \varepsilon$. Then, keeping in mind our assumptions, for a fixed function $u \in C\left(I^{2}\right)$, we get

$\left|(V u)\left(t_{2}, x_{2}\right)-(V u)\left(t_{1}, x_{1}\right)\right|$

$$
\begin{aligned}
& \leq \mid \int_{0}^{t_{2}} \int_{0}^{x_{2}} v\left(t_{2}, s, x_{2}, y, u(s, y)\right) d_{y} g_{2}\left(x_{2}, y\right) d_{s} g_{1}\left(t_{2}, s\right) \\
& \quad-\quad \int_{0}^{t_{1}} \int_{0}^{x_{1}} v\left(t_{1}, s, x_{1}, y, u(s, y)\right) d_{y} g_{2}\left(x_{1}, y\right) d_{s} g_{1}\left(t_{1}, s\right) \mid
\end{aligned}
$$

$$
\begin{aligned}
& \leq \mid \int_{0}^{t_{2}} \int_{0}^{x_{2}} v\left(t_{2}, s, x_{2}, y, u(s, y)\right) d_{y} g_{2}\left(x_{2}, y\right) d_{s} g_{1}\left(t_{2}, g\right) \\
& \quad-\int_{0}^{t_{1}} \int_{0}^{x_{2}} v\left(t_{2}, s, x_{2}, y, u(s, y)\right) d_{y} g_{2}\left(x_{2}, y\right) d_{s} g_{1}\left(t_{2}, s\right) \mid \\
& +\mid \int_{0}^{t_{1}} \int_{0}^{x_{2}} v\left(t_{2}, s, x_{2}, y, u(s, y)\right) d_{y} g_{2}\left(x_{2}, y\right) d_{s} g_{1}\left(t_{2}, s\right) \\
& \quad-\int_{0}^{t_{1}} \int_{0}^{x_{2}} v\left(t_{1}, s, x_{1}, y, u(s, y)\right) d_{y} g_{2}\left(x_{2}, y\right) d_{s} g_{1}\left(t_{2}, s\right) \mid \\
& \quad-\int_{0}^{t_{1}} \int_{0}^{x_{2}} v\left(t_{1}, s, x_{1}, y, u(s, y)\right) d_{y} g_{2}\left(x_{2}, y\right) d_{s} g_{1}\left(t_{2}, s\right) \\
& +\left|\int_{0}^{t_{1}} \int_{0}^{x_{1}} v\left(t_{1}, s, x_{1}, y, u(s, y)\right) d_{y} g_{2}\left(x_{2}, y\right) d_{s} g_{1}\left(t_{2}, s\right)\right|
\end{aligned}
$$


Abstract and Applied Analysis

5

$$
\begin{gathered}
-\int_{0}^{t_{1}} \int_{0}^{x_{1}} v\left(t_{1}, s, x_{1}, y, u(s, y)\right) d_{y} g_{2}\left(x_{1}, y\right) d_{s} g_{1}\left(t_{2}, s\right) \mid \\
+\mid \int_{0}^{t_{1}} \int_{0}^{x_{1}} v\left(t_{1}, s, x_{1}, y, u(s, y)\right) d_{y} g_{2}\left(x_{1}, y\right) d_{s} g_{1}\left(t_{2}, s\right) \\
-\int_{0}^{t_{1}} \int_{0}^{x_{1}} v\left(t_{1}, s, x_{1}, y, u(s, y)\right) d_{y} g_{2}\left(x_{1}, y\right) d_{s} g_{1}\left(t_{1}, s\right) \mid .
\end{gathered}
$$

Further, estimating step by step the terms occurring on the right-hand side of inequality (31), on the basis of Lemmas 1 and 2 , we obtain

$$
\begin{aligned}
& \mid \int_{0}^{t_{2}} \int_{0}^{x_{2}} v\left(t_{2}, s, x_{2}, y, u(s, y)\right) d_{y} g_{2}\left(x_{2}, y\right) d_{s} g_{1}\left(t_{2}, s\right) \\
&-\quad \int_{0}^{t_{1}} \int_{0}^{x_{2}} v\left(t_{2}, s, x_{2}, y, u(s, y)\right) d_{y} g_{2}\left(x_{2}, y\right) d_{s} g_{1}\left(t_{2}, s\right) \mid \\
& \leq\left|\int_{t_{1}}^{t_{2}} \int_{0}^{x_{2}} v\left(t_{2}, s, x_{2}, y, u(s, y)\right) d_{y} g_{2}\left(x_{2}, y\right) d_{s} g_{1}\left(t_{2}, s\right)\right| \\
& \leq \int_{t_{1}}^{t_{2}}\left|\int_{0}^{x_{2}} v\left(t_{2}, s, x_{2}, y, u(s, y)\right) d_{y} g_{2}\left(x_{2}, y\right)\right| d_{s} \\
& \quad \times\left(\bigvee_{p=0}^{s} g_{1}\left(t_{2}, p\right)\right) \\
& \leq \int_{t_{1}}^{t_{2}} \int_{0}^{x_{2}}\left|v\left(t_{2}, s, x_{2}, y, u(s, y)\right)\right| d_{y}\left(\bigvee_{q=0}^{y} g_{2}\left(x_{2}, q\right)\right) d_{s} \\
& \leq K_{2} \phi\left(\|u\|_{C}\right) \bigvee_{s=t_{1}}^{t_{2}} g_{1}\left(t_{2}, s\right) \leq K_{2} \phi\left(\|u\|_{C}\right) N_{1}(\varepsilon), \\
& \leq \quad \int_{t_{1}}^{t_{2}} \int_{0}^{x_{2}} \phi\left(\|u\|_{C}\right) d_{y}\left(\bigvee_{p=0}^{s} g_{1}\left(t_{2}, p\right)\right) \\
&\left.\times g_{2}\left(x_{2}, q\right)\right) d_{s}\left(\bigvee_{p=0} g_{1}\left(t_{2}, p\right)\right)
\end{aligned}
$$

where the function $N_{1}(\varepsilon)$ is defined by (29).

Next, evaluating similarly as above, in view of Lemmas 1 and 2, we have

$$
\begin{aligned}
& \mid \int_{0}^{t_{1}} \int_{0}^{x_{2}} v\left(t_{2}, s, x_{2}, y, u(s, y)\right) d_{y} g_{2}\left(x_{2}, y\right) d_{s} g_{1}\left(t_{2}, s\right) \\
& \quad-\int_{0}^{t_{1}} \int_{0}^{x_{2}} v\left(t_{1}, s, x_{1}, y, u(s, y)\right) d_{y} g_{2}\left(x_{2}, y\right) d_{s} g_{1}\left(t_{2}, s\right) \mid
\end{aligned}
$$

$$
\begin{aligned}
& \leq \mid \int_{0}^{t_{1}} \int_{0}^{x_{2}}\left[v\left(t_{2}, s, x_{2}, y, u(s, y)\right)\right. \\
& \left.\quad-v\left(t_{1}, s, x_{1}, y, u(s, y)\right)\right] \\
& \quad \times d_{y} g_{2}\left(x_{2}, y\right) d_{s} g_{1}\left(t_{2}, s\right) \mid \\
& \leq \int_{0}^{t_{1}} \int_{0}^{x_{2}} \mid v\left(t_{2}, s, x_{2}, y, u(s, y)\right) \\
& \quad-v\left(t_{1}, s, x_{1}, y, u(s, y)\right) \mid d_{y} \\
& \leq \int_{q=0}^{t_{1}} \int_{0}^{x_{2}} \omega_{1,3}(v, \varepsilon) d_{y}\left(\bigvee_{q=0}^{y} g_{2}\left(x_{2}, q\right)\right) d_{s}\left(\bigvee_{p=0}^{s} g_{1}\left(t_{2}, p\right)\right) \\
& \leq \omega_{1,3}(v, \varepsilon)\left(\bigvee_{y=0}^{s} g_{1} g_{1}\left(t_{2}, p\right)\right) \\
& \left.\leq K_{1} K_{2} \omega_{1,3}(v, \varepsilon), y\right)\left(x_{s=0}^{t_{1}} g_{1}\left(t_{2}, p\right)\right)
\end{aligned}
$$

where we denoted

$$
\begin{array}{r}
\omega_{1,3}(v, \varepsilon)=\sup \left\{\left|v\left(t_{2}, s, x_{2}, y, u\right)-v\left(t_{1}, s, x_{1}, y, u\right)\right|:\right. \\
t_{1}, t_{2}, x_{1}, x_{2}, s, y \in I,\left|t_{2}-t_{1}\right| \leq \varepsilon, \\
\left.\left|x_{2}-x_{1}\right| \leq \varepsilon, u \in\left[-\|u\|_{C},\|u\|_{C}\right]\right\} .
\end{array}
$$

Moreover, the constants $K_{1}, K_{2}$ are defined by (24).

Observe that taking into account the fact that the function $v=v(t, s, x, y, u)$ is uniformly continuous on the set $I^{4} \times$ $\left[-\|u\|_{C},\|u\|_{C}\right]$ we deduce that $\omega_{1,3}(v, \varepsilon) \rightarrow 0$ as $\varepsilon \rightarrow 0$.

Further, using the imposed hypotheses, in light of Lemmas 1 and 2, we derive the following estimate:

$$
\begin{aligned}
& \mid \int_{0}^{t_{1}} \int_{0}^{x_{2}} v\left(t_{1}, s, x_{1}, y, u(s, y)\right) d_{y} g_{2}\left(x_{2}, y\right) d_{s} g_{1}\left(t_{2}, s\right) \\
& \quad-\int_{0}^{t_{1}} \int_{0}^{x_{1}} v\left(t_{1}, s, x_{1}, y, u(s, y)\right) d_{y} g_{2}\left(x_{2}, y\right) d_{s} g_{1}\left(t_{2}, s\right) \mid \\
& \leq \int_{0}^{t_{1}}\left|\int_{x_{1}}^{x_{2}} v\left(t_{1}, s, x_{1}, y, u(s, y)\right) d_{y} g_{2}\left(x_{2}, y\right)\right| d_{s} \\
& \quad \times\left(\bigvee_{p=0}^{s} g_{1}\left(t_{2}, p\right)\right) \\
& \leq \int_{0}^{t_{1}} \int_{x_{1}}^{x_{2}}\left|v\left(t_{1}, s, x_{1}, y, u(s, y)\right)\right| d_{y}\left(\bigvee_{q=0}^{y} g_{2}\left(x_{2}, q\right)\right) d_{s} \\
& \quad \times\left(\bigvee_{p=0}^{s} g_{1}\left(t_{2}, p\right)\right)
\end{aligned}
$$




$$
\begin{aligned}
& \leq \phi\left(\|u\|_{C}\right) \int_{0}^{t_{1}} \int_{x_{1}}^{x_{2}} d_{y}\left(\bigvee_{q=0}^{y} g_{2}\left(x_{2}, q\right)\right) d_{s}\left(\bigvee_{p=0}^{s} g_{1}\left(t_{2}, p\right)\right) \\
& \leq \phi\left(\|u\|_{C}\right)\left(\bigvee_{s=0}^{t_{1}} g_{1}\left(t_{2}, s\right)\right)\left(\bigvee_{y=x_{1}}^{x_{2}} g_{2}\left(x_{2}, y\right)\right) \\
& \leq K_{1} \phi\left(\|u\|_{C}\right) N_{2}(\varepsilon),
\end{aligned}
$$

where the constant $K_{1}$ is defined by (24) and the function $\mathrm{N}_{2}(\varepsilon)$ is defined by (29).

Next, using similar reasonings, we arrive at the following estimate:

$$
\begin{aligned}
& \mid \int_{0}^{t_{1}} \int_{0}^{x_{1}} v\left(t_{1}, s, x_{1}, y, u(s, y)\right) d_{y} g_{2}\left(x_{2}, y\right) d_{s} g_{1}\left(t_{2}, s\right) \\
& \quad-\int_{0}^{t_{1}} \int_{0}^{x_{1}} v\left(t_{1}, s, x_{1}, y, u(s, y)\right) d_{y} g_{2}\left(x_{1}, y\right) d_{s} g_{1}\left(t_{2}, s\right) \mid \\
& =\mid \int_{0}^{t_{1}} \int_{0}^{x_{1}} v\left(t_{1}, s, x_{1}, y, u(s, y)\right) d_{y} \\
& \quad \times\left[g_{2}\left(x_{2}, y\right)-g_{2}\left(x_{1}, y\right)\right] d_{s} g_{1}\left(t_{2}, s\right) \mid \\
& \leq \int_{0}^{t_{1}} \int_{0}^{x_{1}}\left|v\left(t_{1}, s, x_{1}, y, u(s, y)\right)\right| d_{y} \\
& \quad \times\left(\bigvee_{q=0}^{y}\left[g_{2}\left(x_{2}, q\right)-g_{2}\left(x_{1}, q\right)\right]\right) d_{s}\left(\bigvee_{p=0}^{s} g_{1}\left(t_{2}, p\right)\right) \\
& \leq \phi\left(\|u\|_{C}\right) \int_{0}^{t_{1}} \int_{0}^{x_{1}} d_{y}\left(\bigvee_{q=0}^{y}\left[g_{2}\left(x_{2}, q\right)-g_{2}\left(x_{1}, q\right)\right]\right) d_{s} \\
& \quad \times\left(\bigvee_{p=0}^{s} g_{1}\left(t_{2}, p\right)\right) \\
& \leq K_{1} \phi\left(\|u\|_{C}\right) M_{2}(\varepsilon), \\
& =\phi\left(\|u\|_{C}\right)\left(\bigvee_{y=0}^{x_{1}}\left[g_{2}\left(x_{2}, y\right)-g_{2}\left(x_{1}, y\right)\right]\right) \bigvee_{s=0}^{t_{1}} g_{1}\left(t_{2}, s\right) \\
& \qquad \bigvee_{y=0}^{x_{1}}\left[g_{2}\left(x_{2}, y\right)-g_{2}\left(x_{1}, y\right)\right] \\
& \leq
\end{aligned}
$$

where the function $M_{2}(\varepsilon)$ was defined in (28).

Now, we estimate the last term appearing on the righthand side of inequality (31). Arguing similarly as above, we get

$$
\begin{aligned}
& \mid \int_{0}^{t_{1}} \int_{0}^{x_{1}} v\left(t_{1}, s, x_{1}, y, u(s, y)\right) d_{y} g_{2}\left(x_{1}, y\right) d_{s} g_{1}\left(t_{2}, s\right) \\
& \quad-\int_{0}^{t_{1}} \int_{0}^{x_{1}} v\left(t_{1}, s, x_{1}, y, u(s, y)\right) d_{y} g_{2}\left(x_{1}, y\right) d_{s} g_{1}\left(t_{1}, s\right) \mid
\end{aligned}
$$

$$
\begin{aligned}
= & \mid \int_{0}^{t_{1}} \int_{0}^{x_{1}} v\left(t_{1}, s, x_{1}, y, u(s, y)\right) d_{y} g_{2}\left(x_{1}, y\right) d_{s} \\
& \times\left[g_{1}\left(t_{2}, s\right)-g_{1}\left(t_{1}, s\right)\right] \mid \\
\leq & \int_{0}^{t_{1}} \int_{0}^{x_{1}}\left|v\left(t_{1}, s, x_{1}, y, u(s, y)\right)\right| d_{y}\left(\bigvee_{q=0}^{y} g_{2}\left(x_{1}, q\right)\right) d_{s} \\
& \times\left(\bigvee_{p=0}^{s}\left[g_{1}\left(t_{2}, p\right)-g_{1}\left(t_{1}, p\right)\right]\right) \\
\leq & \phi\left(\|u\|_{C}\right) \int_{0}^{t_{1}} \int_{0}^{x_{1}} d_{y}\left(\bigvee_{q=0}^{y} g_{2}\left(x_{1}, q\right)\right) d_{s} \\
& \times\left(\bigvee_{p=0}^{s}\left[g_{1}\left(t_{2}, p\right)-g_{1}\left(t_{1}, p\right)\right]\right) \\
= & \phi\left(\|u\|_{C}\right)\left(\bigvee_{y=0}^{x_{1}} g_{2}\left(x_{1}, y\right)\right)\left(\bigvee_{s=0}^{t_{1}}\left[g_{1}\left(t_{2}, p\right)-g_{1}\left(t_{1}, s\right)\right]\right) \\
\leq & K_{2} \phi\left(\|u\|_{C}\right) M_{1}(\varepsilon),
\end{aligned}
$$

where the constant $K_{2}$ and the function $M_{1}(\varepsilon)$ were defined in (24) and (28), respectively.

Now, linking estimates (31)-(33) and (34)-(37) as well as taking into account the properties of the functions $M_{i}=$ $M_{i}(\varepsilon)$ and $N_{i}=N_{i}(\varepsilon)(i=1,2)$ and the comment given after estimate (33), we conclude that the operator $V$ transforms the space $C\left(I^{2}\right)$ into itself.

On the other hand, keeping in mind Lemma 4 we infer that the operator $F$ defined in (30) transforms also the space $C\left(I^{2}\right)$ into itself. Consequently we obtain that the operator $Q$ defined in (30) is a self-mapping of the space $C\left(I^{2}\right)$.

In the sequel we show that the operator $Q$ is continuous on the space $C\left(I^{2}\right)$. To this end let us first observe that in view of the properties of the superposition operator $F$ expressed in Lemma 4 it is sufficient to show that the operator $V$ defined by (30) is continuous on $C\left(I^{2}\right)$. To prove this fact fix arbitrarily $\varepsilon>0$ and $u \in C\left(I^{2}\right)$. Further, take an arbitrary function $w \in C\left(I^{2}\right)$ with $\|u-w\|_{C} \leq \varepsilon$. Then, keeping in mind Lemma 1 , for arbitrarily fixed $t, x \in I$, we obtain

$$
\begin{aligned}
& |(V u)(t, x)-(V w)(t, x)| \\
& \leq \int_{0}^{t} \int_{0}^{x}|v(t, s, x, y, u(s, y))-v(t, s, x, y, w(s, y))| d_{y} \\
& \quad \times\left(\bigvee_{q=0}^{y} g_{2}(x, q)\right) d_{s}\left(\bigvee_{p=0}^{s} g_{1}(t, p)\right) .
\end{aligned}
$$

Next, let us denote

$$
P=\|u\|_{C}+\varepsilon
$$


and let us define

$$
\begin{gathered}
\omega_{P}(v, \varepsilon)=\sup \left\{\left|v\left(t, s, x, y, u_{1}\right)-v\left(t, s, x, y, u_{2}\right)\right|:\right. \\
(t, s) \in \Delta_{1},(x, y) \in \Delta_{2}, \\
\left.u_{1} u_{2} \in[-P, P],\left|u_{1}-u_{2}\right| \leq \varepsilon\right\} .
\end{gathered}
$$

Then, from (38) we derive the following estimates:

$$
\begin{aligned}
& |(V u)(t, x)-(V w)(t, x)| \\
& \leq \int_{0}^{t} \int_{0}^{x} \omega_{P}(v, \varepsilon) d_{y}\left(\bigvee_{q=0}^{y} g_{2}(x, q)\right) d_{s}\left(\bigvee_{p=0}^{s} g_{1}(t, p)\right) \\
& \leq \omega_{P}(v, \varepsilon)\left(\bigvee_{y=0}^{x} g_{2}(x, y)\right)\left(\bigvee_{s=0}^{t} g_{1}(t, s)\right) \\
& \leq K_{1} K_{2} \omega_{P}(v, \varepsilon) .
\end{aligned}
$$

Hence, in view of the uniform continuity of the function $v$ on the set $\Delta_{1} \times \Delta_{2} \times[-P, P]$, we infer that the operator $V$ is continuous on the space $C\left(I^{2}\right)$. According to the above remark this implies that the operator $Q$ is continuous on the space $C\left(I^{2}\right)$.

In what follows let us fix an arbitrary function $u \in C\left(I^{2}\right)$. Utilizing the imposed assumptions and Lemmas 1 and 2, for fixed $t, x \in I$, we obtain

$$
\begin{aligned}
& |(Q u)(t, x)| \\
& \leq|h(t, x)|+|f(t, x, u(t, x))| \\
& \quad \times \int_{0}^{t}\left|\int_{0}^{x} v(t, s, x, y, u(s, y)) d_{y} g_{2}(x, y)\right| d_{s} \\
& \quad \times\left(\bigvee_{p=0}^{s} g_{1}(t, p)\right) \\
& \leq\|h\|_{C}+[|f(t, x, u(t, x))-f(t, x, 0)|+|f(t, x, 0)|] \\
& \quad \times \int_{0}^{t} \int_{0}^{x}|v(t, s, x, y, u(s, y))| d_{y}\left(\bigvee_{q=0}^{y} g_{2}(x, q)\right) d_{s} \\
& \quad \times\left(\bigvee_{p=0}^{s} g_{1}(t, p)\right) \\
& \leq\|h\|_{C}+[k|u(t, x)|+|f(t, x, 0)|] \\
& \quad \times \int_{0}^{t} \int_{0}^{x} \phi(|u(s, y)|) d_{y}\left(\bigvee_{q=0}^{y} g_{2}(x, q)\right) d_{s} \\
& \quad \times\left(\bigvee_{p=0}^{s} g_{1}(t, p)\right) \\
& \leq\|h\|_{C}+\left(k\|u\|_{C}+F_{1}\right) \phi\left(\|u\|_{C}\right)
\end{aligned}
$$

$$
\begin{aligned}
& \times \int_{0}^{t} \int_{0}^{x} d_{y}\left(\bigvee_{q=0}^{y} g_{2}(x, q)\right) d_{s}\left(\bigvee_{p=0}^{s} g_{1}(t, p)\right) \\
= & \|h\|_{C}+\left(k\|u\|_{C}+F_{1}\right) \phi\left(\|u\|_{C}\right)\left(\bigvee_{y=0}^{x} g_{2}(x, y)\right) \\
& \times\left(\bigvee_{s=0}^{t} g_{1}(t, s)\right) .
\end{aligned}
$$

Hence, in light of Corollary 9, we obtain the following estimate:

$$
|(Q u)(t, x)| \leq\|h\|_{C}+\left(k\|u\|_{C}+F_{1}\right) K_{1} K_{2} \phi\left(\|u\|_{C}\right) .
$$

Consequently, we get

$$
\|Q u\|_{C} \leq\|h\|_{C}+\left(k\|u\|_{C}+F_{1}\right) K_{1} K_{2} \phi\left(\|u\|_{C}\right) .
$$

Now, taking into account assumption (viii), from estimate (44), we derive that there exists a number $r_{0}>0$ such that $Q$ transforms the ball $B_{r_{0}}$ into itself and $k K_{1} K_{2} \phi\left(r_{0}\right)<1$.

Next, let us take a nonempty subset $X$ of the ball $B_{r_{0}}$ and choose arbitrarily a function $u \in X$. Then, for a fixed $\varepsilon>0$ and for arbitrary $\left(t_{1}, x_{1}\right),\left(t_{2}, x_{2}\right) \in I^{2}$ such that $t_{1} \leq t_{2}, x_{1}$ $\leq x_{2}$ (cf. Remark 11) and $t_{2}-t_{1} \leq \varepsilon, x_{2}-x_{1} \leq \varepsilon$, using the standard tools, we get

$$
\begin{aligned}
\left|(Q u)\left(t_{2}, x_{2}\right)-(Q u)\left(t_{1}, x_{1}\right)\right| \\
\leq\left|h\left(t_{2}, x_{2}\right)-h\left(t_{1}, x_{1}\right)\right| \\
\quad+\left|(F u)\left(t_{2}, x_{2}\right)(V u)\left(t_{2}, x_{2}\right)-(F u)\left(t_{2}, x_{2}\right)(V u)\left(t_{1}, x_{1}\right)\right| \\
\quad+\left|(F u)\left(t_{2}, x_{2}\right)(V u)\left(t_{1}, x_{1}\right)-(F u)\left(t_{1}, x_{1}\right)(V u)\left(t_{1}, x_{1}\right)\right| \\
\leq \omega(h, \varepsilon)+\left|(F u)\left(t_{2}, x_{2}\right)\right|\left|(V u)\left(t_{2}, x_{2}\right)-(V u)\left(t_{1}, x_{1}\right)\right| \\
\quad+\left|(V u)\left(t_{1}, x_{1}\right)\right|\left|(F u)\left(t_{2}, x_{2}\right)-(F u)\left(t_{1}, x_{1}\right)\right| \\
\leq \omega(h, \varepsilon)+\left[\left|f\left(t_{2}, x_{2}, u\left(t_{2}, x_{2}\right)\right)-f\left(t_{2}, x_{2}, 0\right)\right|\right. \\
\left.\quad+\left|f\left(t_{2}, x_{2}, 0\right)\right|\right] \\
\quad \times\left\{\left|(V u)\left(t_{2}, x_{2}\right)-(V u)\left(t_{1}, x_{1}\right)\right|\right\}+\left|(V u)\left(t_{1}, x_{1}\right)\right| \\
\quad \times\left\{\left|f\left(t_{2}, x_{2}, u\left(t_{2}, x_{2}\right)\right)-f\left(t_{2}, x_{2}, u\left(t_{1}, x_{1}\right)\right)\right|\right. \\
\left.\quad+\left|f\left(t_{2}, x_{2}, u\left(t_{1}, x_{1}\right)\right)-f\left(t_{1}, x_{1}, u\left(t_{1}, x_{1}\right)\right)\right|\right\} \\
\leq \omega(h, \varepsilon)+\left[k\left|u\left(t_{2}, x_{2}\right)\right|+\left|f\left(t_{2}, x_{2}, 0\right)\right|\right] \\
\quad \times\left\{\left|(V u)\left(t_{2}, x_{2}\right)-(V u)\left(t_{1}, x_{1}\right)\right|\right\} \\
\quad+\left|(V u)\left(t_{1}, x_{1}\right)\right|\left\{k\left|u\left(t_{2}, x_{2}\right)-u\left(t_{1}, x_{1}\right)\right|+\bar{\omega}(f, \varepsilon)\right\} \\
\leq \omega(h, \varepsilon)+\left(k\|u\|_{C}+F_{1}\right)\left\{\left|(V u)\left(t_{2}, x_{2}\right)-(V u)\left(t_{1}, x_{1}\right)\right|\right\} \\
+\left|(V u)\left(t_{1}, x_{1}\right)\right|(k \omega(u, \varepsilon)+\bar{\omega}(f, \varepsilon)),
\end{aligned}
$$


where we denoted

$$
\begin{array}{r}
\bar{\omega}(f, \varepsilon)=\sup \left\{\left|f\left(t_{2}, x_{2}, u\right)-f\left(t_{1}, x_{1}, u\right)\right|:\right. \\
t_{1}, t_{2}, x_{1}, x_{2} \in I, u \in[-r, r], \\
\left.\left|t_{2}-t_{1}\right| \leq \varepsilon,\left|x_{2}-x_{1}\right| \leq \varepsilon\right\} .
\end{array}
$$

Further, based on estimates (31)-(33) and (35)-(37) we obtain

$$
\begin{aligned}
& \left|(V u)\left(t_{2}, x_{2}\right)-(V u)\left(t_{1}, x_{1}\right)\right| \\
& \leq K_{2} \phi\left(\|u\|_{C}\right) \bigvee_{s=t_{1}}^{t_{2}} g_{1}\left(t_{2}, s\right) \\
& \quad+K_{1} K_{2} \omega_{1,3}(v, \varepsilon)+K_{1} \phi\left(\|u\|_{C}\right) \bigvee_{y=x_{1}}^{x_{2}} g_{2}\left(x_{2}, y\right) \\
& \quad+K_{1} \phi\left(\|u\|_{C}\right) M_{2}(\varepsilon)+K_{2} \phi\left(\|u\|_{C}\right) M_{1}(\varepsilon) .
\end{aligned}
$$

Now, from (47) and (29) we derive the following estimate:

$$
\begin{aligned}
\omega(V u, \varepsilon) \leq & K_{2} \phi\left(r_{0}\right) N_{1}(\varepsilon)+K_{1} \phi\left(r_{0}\right) N_{2}(\varepsilon) \\
& +K_{1} \phi\left(r_{0}\right) M_{2}(\varepsilon)+K_{2} \phi\left(r_{0}\right) M_{1}(\varepsilon) .
\end{aligned}
$$

The above estimate yields the following one:

$$
\begin{aligned}
\omega(V X, \varepsilon) \leq & K_{2} \phi\left(r_{0}\right) N_{1}(\varepsilon)+K_{1} \phi\left(r_{0}\right) N_{2}(\varepsilon) \\
& +K_{1} K_{2} \omega_{1,3}(v, \varepsilon)+K_{1} \phi\left(r_{0}\right) M_{2}(\varepsilon) \\
& +K_{2} \phi\left(r_{0}\right) M_{1}(\varepsilon) .
\end{aligned}
$$

On the other hand, using our assumptions and applying Lemmas 1 and 2, we get

$$
\begin{aligned}
& \left|(V u)\left(t_{1}, x_{1}\right)\right| \\
& \leq \int_{0}^{t_{1}} \int_{0}^{x_{1}}\left|v\left(t_{1}, s, x_{1}, y, u(s, y)\right)\right| d_{y}\left(\bigvee_{q=0}^{y} g_{2}\left(x_{1}, q\right)\right) d_{s} \\
& \quad \times\left(\bigvee_{p=0}^{s} g_{1}\left(t_{1}, p\right)\right) \\
& \leq \phi\left(\|u\|_{C}\right) \int_{0}^{t_{1}} \int_{0}^{x_{1}} d_{y}\left(\bigvee_{q=0}^{y} g_{2}\left(x_{1}, q\right)\right) d_{s}\left(\bigvee_{p=0}^{s} g_{1}\left(t_{1}, p\right)\right) \\
& \leq \phi\left(r_{0}\right)\left(\bigvee_{y=0}^{x_{1}} g_{2}\left(x_{1}, y\right)\right)\left(\bigvee_{s=0}^{t_{1}} g_{1}\left(t_{1}, p\right)\right) \leq K_{1} K_{2} \phi\left(r_{0}\right) .
\end{aligned}
$$

Finally, combining estimates (45), (49), and (50), we get

$$
\begin{aligned}
\omega(Q X, \varepsilon) \leq & \omega(h, \varepsilon) \\
+ & \\
+\left(k r_{0}+F_{1}\right) & \left\{K_{2} \phi\left(r_{0}\right) N_{1}(\varepsilon)+K_{1} \phi\left(r_{0}\right) N_{2}(\varepsilon)\right. \\
& +K_{1} K_{2} \omega_{1,3}(v, \varepsilon)+K_{1} \phi\left(r_{0}\right) M_{2}(\varepsilon) \\
& \left.+K_{2} \phi\left(r_{0}\right) M_{1}(\varepsilon)\right\} \\
& +K_{1} K_{2} \phi\left(r_{0}\right)\{k \omega(X, \varepsilon)+\bar{\omega}(f, \varepsilon)\} .
\end{aligned}
$$

Hence, taking into account the properties of the functions $M_{i}, N_{i}(i=1,2)$ and the functions $\varepsilon \rightarrow \omega(h, \varepsilon), \varepsilon \rightarrow$ $\omega_{1,3}(v, \varepsilon)$, and $\varepsilon \rightarrow \bar{\omega}(f, \varepsilon)$, we obtain

$$
\omega_{0}(Q X) \leq k K_{1} K_{2} \phi\left(r_{0}\right) \omega_{0}(X) .
$$

Keeping in mind assumption (viii) and Theorem 3 and taking into account formula (11), from (52) we deduce that there exists at least one function $u=u(t, x)$ belonging to the ball $B_{r_{0}}$ which is a solution of (16).

The proof is complete.

Remark 11. In considerations conducted in the above proof, taking two points $\left(t_{1}, x_{1}\right),\left(t_{2}, x_{2}\right) \in I^{2}$, we assumed that $t_{1} \leq$ $t_{2}$ and $x_{1} \leq x_{2}$.

Observe that all possible cases can be always converted to that indicated above. For example, if we assume that $t_{1} \leq t_{2}$ and $x_{1}>x_{2}$, then, taking an arbitrary function $w=w(t, x, z)$ with real variables ( $z$ denotes an arbitrary real number), we get

$$
\begin{aligned}
& \left|w\left(t_{2}, x_{2}, z\right)-w\left(t_{1}, x_{1}, z\right)\right| \\
& \leq\left|w\left(t_{2}, x_{2}, z\right)-w\left(t_{1}, x_{2}, z\right)\right| \\
& \quad+\left|w\left(t_{1}, x_{2}, z\right)-w\left(t_{1}, x_{1}, z\right)\right| \\
& =\left|w\left(t_{2}, x_{2}, z\right)-w\left(t_{1}, x_{2}, z\right)\right| \\
& \quad+\left|w\left(t_{1}, x_{1}, z\right)-w\left(t_{1}, x_{2}, z\right)\right| .
\end{aligned}
$$

Now, we can repeat all estimates of the above proof under requirements concerning the choice of the points $\left(t_{1}, x_{1}\right)$, $\left(t_{2}, x_{2}\right)$.

\section{Applications to Functional Integral Equations of Fractional Order and to Other Types of Functional Integral Equations}

We start with providing some facts concerning assumption (v) imposed in investigations conducted in the preceding section (cf. also [9]). At the beginning we formulate a condition which is handy and convenient in applications and which guarantees that the functions $g_{1}, g_{2}$ appearing in (16) satisfy assumption $(\mathrm{v})$.

In order to formulate the announced condition assume (as we have done previously) that $g_{i}(w, z)=g_{i}: \Delta_{i} \rightarrow \mathbb{R}$ is a given function $(i=1,2)$. Further we assume that the function $g_{i}(w, z)(i=1,2)$ satisfies the following condition:

$\left(\mathrm{v}^{\prime}\right)$ for arbitrary $w_{1}, w_{2} \in I, w_{1}<w_{2}$, the function $z \rightarrow g_{i}\left(w_{2}, z\right)-g_{i}\left(w_{1}, z\right)$ is monotone on the interval $\left[0, w_{1}\right](i=1,2)$.

From results proved in [9] the following lemma immediately follows.

Lemma 12. Suppose the function $g_{i}=g_{i}(w, z)$ satisfies assumptions (iii), $\left(v^{\prime}\right)$, and (vi) for $i=1,2$. Then $g_{i}$ satisfies assumption $(v)(i=1,2)$. 
Indeed, in the case when $g_{i}$ is nonincreasing, this result was proved as Theorem 3 in [9], while the case when $g_{i}$ is nondecreasing is covered by Theorem 5 in $[9](i=1,2)$.

Further, based on results obtained in [9] we provide two examples of functions $g_{i}=g_{i}(w, z)$ satisfying assumption $\left(\mathrm{v}^{\prime}\right)$ and being essential in our considerations.

Example 13. Let us fix $i(i=1,2)$ and take the function $g_{i}(w, z)=g_{i}: \Delta_{i} \rightarrow \mathbb{R}$ defined by the formula

$$
g_{i}(w, z)=\frac{1}{\alpha}\left[w^{\alpha}-(w-z)^{\alpha}\right],
$$

where $\alpha$ is a fixed number from the interval $(0,1)$. If we fix arbitrary numbers $w_{1}, w_{2} \in I$ such that $w_{1}<w_{2}$, then it is easily seen that the function $z \rightarrow g_{i}\left(w_{2}, z\right)-g_{i}\left(w_{1}, z\right)$ is nonincreasing on the interval $\left[0, w_{1}\right]$. This means that $g_{i}$ satisfies assumption $\left(\mathrm{v}^{\prime}\right)$. Moreover, we can verify that the function $g_{i}(w, z)$ satisfies also assumptions (iii), (iv), and (vi).

Example 14. Similarly as above fix $i \in\{1,2\}$. Consider the function $g_{i}(w, z)=g_{i}: \Delta_{i} \rightarrow \mathbb{R}$ defined by the formula

$$
g_{i}(w, z)= \begin{cases}w \ln \frac{w+z}{w} & \text { for } 0<z \leq w \leq 1 \\ 0 & \text { for } w=0\end{cases}
$$

Using the standard methods of mathematical analysis (cf. [9]) it can be easily shown that the function $z \rightarrow g_{i}\left(w_{2}, z\right)-$ $g_{i}\left(w_{1}, z\right)$ is nondecreasing on the interval $\left[0, w_{1}\right]$ for $w_{1}<w_{2}$ and satisfies assumptions (iii), (iv), and (vi) formulated in the preceding section.

In what follows we will consider the fractional integral equation with functions involved depending on two variables, which has the form

$$
\begin{aligned}
u(t, x)= & h(t, x) \\
& +\frac{f(t, x, u(t, x))}{\Gamma(\alpha) \Gamma(\beta)} \int_{0}^{t} \int_{0}^{x} \frac{v(t, s, x, y, u(s, y))}{(t-s)^{1-\alpha}(x-y)^{1-\beta}} d s d y,
\end{aligned}
$$

where $t, x \in I$ and $\alpha, \beta$ are fixed numbers from the interval $(0,1)$. Moreover, the symbol $\Gamma(\gamma)$ indicates the gamma function.

Let us mention that (56) represents the so-called partial singular integral equation of Volterra type in two variables. Recently, equations of such a type were intensively investigated in some papers [11-16].

Obviously, (56) creates a generalization of the classical Volterra integral equation of fractional order in one variable which is studied in several papers and monographs and finds numerous applications (cf. [1-10, 23-27] and references therein).

Now, we show that the functional integral equation of fractional orders (56) can be treated as a particular case of the Volterra-Stieltjes functional integral equation (16) studied in Section 3.
In fact, take the functions $g_{i}(w, z)$ considered in Example 13, which have the form

$$
\begin{gathered}
g_{1}(t, s)=\frac{1}{\alpha}\left[t^{\alpha}-(t-s)^{\alpha}\right], \\
g_{2}(x, y)=\frac{1}{\beta}\left[x^{\beta}-(x-y)^{\beta}\right],
\end{gathered}
$$

for $(t, s) \in \Delta_{1}$ and $(x, y) \in \Delta_{2}$.

Then, it can be easily seen that (56) can be written in the form of (16). Thus, we can apply Theorem 10 in order to obtain an existence result concerning (56).

To formulate such a result let us first calculate the constants $K_{1}, K_{2}$ from Corollary 9. Indeed, we have (cf. [9])

$$
\begin{aligned}
& K_{1}=\sup \left\{\bigvee_{z=0}^{w} g_{1}(w, z): w \in I\right\}=\frac{1}{\alpha}, \\
& K_{2}=\sup \left\{\bigvee_{z=0}^{w} g_{2}(w, z): w \in I\right\}=\frac{1}{\beta} .
\end{aligned}
$$

Now, we present the above announced result.

Theorem 15. Assume that the function $h$ involved in (56) satisfies assumption (i) and the function $v=v(t, s, x, y, u)$ satisfies assumption (vii) of Theorem 10. Moreover, one assumes that the following condition is satisfied.

(viii') There exists a positive solution $r_{0}$ of the inequality

$$
\|h\|_{C}+\frac{k r+F_{1}}{\Gamma(\alpha+1) \Gamma(\beta+1)} \phi(r) \leq r
$$

$$
\text { such that } k \phi\left(r_{0}\right)<\Gamma(\alpha+1) \Gamma(\beta+1) \text {. }
$$

Then there exists at least one solution $u=u(t, x)$ of $(56)$ in the space $C\left(I^{2}\right)$, belonging to the ball $B_{r_{0}}$.

Remark 16. In the above conducted calculations we used the well-known formula $\delta \Gamma(\delta)=\Gamma(\delta+1)$ (cf. [28]).

In what follows we consider the functional integral equation of the so-called Volterra-Chandrasekhar type in two variables having the form

$$
\begin{aligned}
& u(t, x)= h(t, x) \\
&+f(t, x, u(t, x)) \int_{0}^{t} \int_{0}^{x} \frac{t x}{(t+s)(x+y)} \\
& \quad \times v(t, s, x, y, u(s, y)) d s d y
\end{aligned}
$$

for $t, x \in I$. We refer to $[4,9,29,30]$ for the case of the Chandrasekhar and Volterra-Chandrasekhar equations in one variable. 
Observe that taking the function $g=g_{i}=g_{i}(y, z)(i=$ $1,2)$ appearing in Example 14 we can represent $(60)$ in the form (16). Obviously, in this case we take

$$
\begin{aligned}
& g_{1}(t, s)= \begin{cases}t \ln \frac{t+s}{t} & \text { for } 0<s \leq t \leq 1 \\
0 & \text { for } t=0,\end{cases} \\
& g_{2}(x, y)= \begin{cases}x \ln \frac{x+y}{x} & \text { for } 0<y \leq x \leq 1 \\
0 & \text { for } x=0 .\end{cases}
\end{aligned}
$$

Further, using the fact that the function $g$ satisfies assumption $\left(\mathrm{v}^{\prime}\right)$, we get

$$
\sup \left\{\bigvee_{z=0}^{w} g(w, z): w \in I\right\}=\sup \{g(w, w): w \in I\}=\ln 2
$$

Now, we can formulate an existence theorem concerning (60).

Theorem 17. Assume that the function $h$ appearing in (60) satisfies assumption (i), the function $f=f(t, x, u)$ satisfies assumption (ii), and the function $v=v(t, s, x, y, u)$ satisfies assumption (vii) of Theorem 10. Apart from this one assumes that the following condition is satisfied.

(viii") There exists a positive solution $r_{0}$ of the inequality

$$
\|h\|_{C}+\left(k r+F_{1}\right) \phi(r) \ln ^{2} 2 \leq r
$$

such that $k \phi\left(r_{0}\right) \ln ^{2} 2<1$.

Then there exists at least one solution $u=u(t, x)$ of $(60)$ in the space $C\left(I^{2}\right)$, belonging to the ball $B_{r_{0}}$.

Finally, we consider the functional integral equation having the form linking equations (56) and (60), that is, the following integral equation:

$$
\begin{aligned}
u(t, x)= & h(t, x)+\frac{f(t, x, u(t, x))}{\Gamma(\alpha)} \\
& \times \int_{0}^{t} \int_{0}^{x} \frac{x v(t, s, x, y, u(s, y))}{(x+y)(t-s)^{1-\alpha}} d s d y,
\end{aligned}
$$

where, as above, $\alpha$ is a fixed number in the interval $(0,1)$ and $\Gamma(\alpha)$ denotes the gamma function.

An existence theorem concerning (64) can be formulated almost in the same way as Theorems 15 and 17 . We need only to replace assumptions (viii') and (viii") by the following one.

(viii) There exists a positive solution $r_{0}$ of the following inequality:

$$
\|h\|_{C}+\frac{\left(k r+F_{1}\right) \ln 2}{\Gamma(\alpha+1)} \phi(r) \leq r
$$

such that $k \phi\left(r_{0}\right) \ln 2<\Gamma(\alpha+1)$.

We omit other details.

\section{Final Remarks concerning Possible Generalizations}

In this section we focus briefly on possible generalizations of results presented in previous sections.

First of all let us notice that instead of the functional integral equation of Volterra-Stieltjes type in two variables (16) we can consider the general case of the functional integral equation of Volterra-Stieltjes type in $n$ variables which has the form

$$
u(t)=h(t)+f(t, u(t))(V u)(t),
$$

where we denoted

$$
t=\left(t_{1}, t_{2}, \ldots, t_{n}\right)
$$

$(V u)(t)$

$$
\begin{aligned}
& =\int_{0}^{t_{1}} \int_{0}^{t_{2}} \ldots \int_{0}^{t_{n}} v\left(t_{1}, s_{1}, t_{2}, s_{2}, \ldots, t_{n}, s_{n}, u\left(s_{1}, s_{2}, \ldots s_{n}\right)\right) \\
& \times d_{s_{1}} g_{1}\left(t_{1}, s_{1}\right) d_{s_{2}} g_{2}\left(t_{2}, s_{2}\right) \\
& \cdots d_{s_{n}} g_{n}\left(t_{n}, s_{n}\right) \text {. }
\end{aligned}
$$

We assume here that $t=\left(t_{1}, t_{2}, \ldots, t_{n}\right) \in I^{n}$ and the functions involved in (66) satisfy assumptions similar to those formulated in Theorem 10. For example, $g_{i}: \Delta_{i} \rightarrow \mathbb{R}$ is a continuous function on the triangle $\Delta_{i}=\left\{\left(t_{i}, s_{i}\right): 0 \leq\right.$ $\left.s_{i} \leq t_{i} \leq 1\right\}$ for $i=1,2, \ldots, n$. Apart from this the function $g_{i}$ satisfies assumptions (iv)-(vi) of Theorem $10(i=1,2, \ldots, n)$. Regarding the function $v=v\left(t_{1}, s_{1}, t_{2}, s_{2}, \ldots, t_{n}, s_{n}, u\right)$ we assume that $v: \Delta_{1} \times \Delta_{2} \times \cdots \times \Delta_{n} \times \mathbb{R} \rightarrow \mathbb{R}$ is continuous and such that

$$
\left|v\left(t_{1}, s_{1}, t_{2}, s_{2}, \ldots, t_{n}, s_{n}, u\right)\right| \leq \phi(u)
$$

for all $\left(t_{i}, s_{i}\right) \in \Delta_{i}(i=1,2, \ldots, n)$ and for each $u \in \mathbb{R}$, where (similarly as in assumption (vii)) $\phi: \mathbb{R}_{+} \rightarrow \mathbb{R}_{+}$is a nondecreasing function.

Other assumptions concerning (66), that is, assumptions (i), (ii), and (viii), can be easily adapted to our case.

Let us mention that we can also investigate equations considered precedingly, that is, (16), (56), (60), and (64), in the case when we replace the bounded interval $I$ by an unbounded interval, for example, by $\mathbb{R}_{+}$. Such a situation was considered in [10] in the case of the functional integral equation of Volterra-Stieltjes type in one variable. Obviously, even in the case of one variable considered in [10], investigations are very extensive and complicated. On the other hand, those investigations allow us to study some qualitative aspects concerning solutions of the equation in question, such as asymptotic behaviour, stability, and asymptotic stability.

One can expect that in the case of (16), (56), and so forth, we can also investigate qualitative aspects of solutions of those equations. Investigations of such a type will appear elsewhere. 


\section{Conflict of Interests}

The authors declare that there is no conflict of interests in the submitted paper.

\section{Acknowledgment}

This work was funded by the Deanship of Scientific Research (DSR), King Abdulaziz University, Jeddah, under Grant no. (363-001-D1434). The authors, therefore, acknowledge with thanks DSR technical and financial support.

\section{References}

[1] D. Baleanu, K. Diethelm, E. Scalas, and J. J. Trujillo, Fractional Calculus: Models and Numerical Methods, World Scientific, New York, NY, USA, 2012.

[2] K. Diethelm, The Analysis of Fractional Differential Equations, Lecture Notes in Mathematics, Springer, Berlin, Germany, 2010.

[3] R. Hilfer, Applications of Fractional Calculus in Physics, World Scientific, River Edge, NJ, USA, 2000.

[4] V. E. Tarasov, Fractional Dynamics: Applications of Fractional Calculus to Dynamics of Particles, Fields and Media, Nonlinear Physical Science, Springer, Heidelberg, Germany; Higher Education Press, Beijing, China, 2010.

[5] N. T. Dung, "Fractional stochastic differential equations with applications to finance," Journal of Mathematical Analysis and Applications, vol. 397, no. 1, pp. 334-348, 2013.

[6] S. Abbas, M. Benchohra, and G. M. N’Guérékata, Topic in Fractional Differential Equations, vol. 27 of Developments in Mathematics, Springer, New York, NY, USA, 2012.

[7] A. A. Kilbas, H. M. Srivastava, and J. J. Trujillo, Theory and Applications of Fractional Differential Equations, vol. 204 of North-Holland Mathematics Studies, Elsevier Science B.V., Amsterdam, The Netherlands, 2006.

[8] I. Podlubny, Fractional Differential Equations, vol. 198 of Mathematics in Science and Engineering, Academic Press, San Diego, Calif, USA, 1999.

[9] J. Banaś and T. Zajaç, "A new approach to the theory of functional integral equations of fractional order," Journal of Mathematical Analysis and Applications, vol. 375, no. 2, pp. 375387, 2011.

[10] T. Zajac, "Solvability of fractional integral equations on an unbounded interval through the theory of Volterra-Stieltjes integral equations," Zeitschrift für Analysis und ihre Anwendungen, vol. 33, no. 1, pp. 65-85, 2014.

[11] S. Abbas, D. Baleanu, and M. Benchohra, "Global attractivity for fractional order delay partial integro-differential equations," Advances in Difference Equations, vol. 2012, article 62, 2012.

[12] S. Abbas, M. Benchohra, and J. J. Nieto, "Global attractivity of solutions for nonlinear fractional order Riemann-Liouville Volterra-Stieltjes partial integral equations," Electronic Journal of Qualitative Theory of Differential Equations, no. 81, pp. 1-15, 2012.

[13] S. Abbas and M. Benchohra, "Existence and stability of nonlinear, fractional order Riemann-Liouville Volterra-Stieltjes multidelay integral equations," Journal of Integral Equations and Applications, vol. 25, no. 2, pp. 143-158, 2013.
[14] S. Abbas and M. Benchohra, "Fractional order integral equations of two independent variables," Applied Mathematics and Computation, vol. 227, pp. 755-761, 2014.

[15] A. N. Vityuk and A. V. Golushkov, "Existence of solutions of systems of partial differential equations of fractional order," Nonlinear Oscillations, vol. 7, no. 3, pp. 328-335, 2004.

[16] B. Ahmad, J. J. Nieto, A. Alsaedi, and H. Al-Hutami, "Existence of solutions for nonlinear fractional $q$-difference integral equations with two fractional orders and nonlocal four-point boundary conditions," Journal of the Franklin Institute, vol. 351, no. 5, pp. 2890-2909, 2014.

[17] J. Appell, J. Banaś, and N. Merentes, Bounded Variation and Around, vol. 17 of Series in Nonlinear Analysis and Applications, Walter de Gruyter, Berlin, Germany, 2014.

[18] W. Rudin, Real and Complex Analysis, McGraw-Hill, New York, NY, USA, 1970.

[19] J. Banaś and K. Goebel, Measures of Noncompactness in Banach Spaces, vol. 60 of Lecture Notes in Pure and Applied Mathematics, Marcel Dekker, New York, NY, USA, 1980.

[20] R. R. Akhmerov, M. I. Kamenskiü, A. S. Potapov, A. E. Rodkina, and B. N. Sadovskii, Measures of Noncompactness and Condensing Operators, vol. 55 of Operator Theory: Advances and Applications, Birkhäuser, Basel, Switzerland, 1992.

[21] M. A. Toledano, T. D. Benavides, and G. L. Acedo, Measures of Noncompactness in Metric Fixed Point Theory, vol. 99 of Operator Theory: Advances and Applications, Birkhäuser, Basel, Switzerland, 1997.

[22] J. Appell and P. P. Zabrejko, Nonlinear Superposition Operators, vol. 95 of Cambridge Tracts in Mathematics, Cambridge University Press, Cambridge, UK, 1990.

[23] H. M. Srivastava and R. K. Saxena, "Operators of fractional integration and their applications," Applied Mathematics and Computation, vol. 118, no. 1, pp. 1-52, 2001.

[24] M. A. Darwish, "Nondecreasing solutions of a fractional quadratic integral equation of Urysohn-Volterra type," Dynamic Systems and Applications, vol. 20, no. 4, pp. 423-438, 2011.

[25] M. A. Darwish and S. K. Ntouyas, "On a quadratic fractional Hammerstein-Volterra integral equation with linear modification of the argument," Nonlinear Analysis: Theory, Methods \& Applications, vol. 74, no. 11, pp. 3510-3517, 2011.

[26] S. Abbas, M. Benchohra, and J. Henderson, "Global asymptotic stability of solutions of nonlinear quadratic Volterra integral equations of fractional order," Communications on Applied Nonlinear Analysis, vol. 19, no. 1, pp. 79-89, 2012.

[27] J. Caballero, M. A. Darwish, and K. Sadarangani, "Solvability of a fractional hybrid initial value problem with supremum by using measures of noncompactness in Banach algebras," Applied Mathematics and Computation, vol. 224, pp. 553-563, 2013.

[28] G. M. Fichtenholz, Differential and Integral Calculus, vol. 2, Wydawnictwo Naukowe PWN, Warsaw, Poland, 2007 (Polish).

[29] I. K. Argyros, "On a class of quadratic integral equations with perturbation," Functiones et Approximatio Commentarii Mathematici, vol. 20, pp. 51-63, 1992.

[30] S. Chandrasekhar, Radiative Transfer, Oxford University Press, London, UK, 1950. 


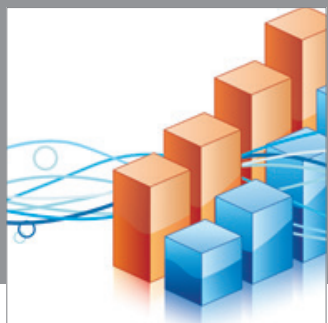

Advances in

Operations Research

mansans

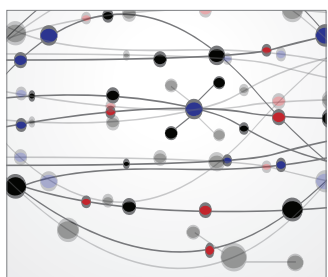

The Scientific World Journal
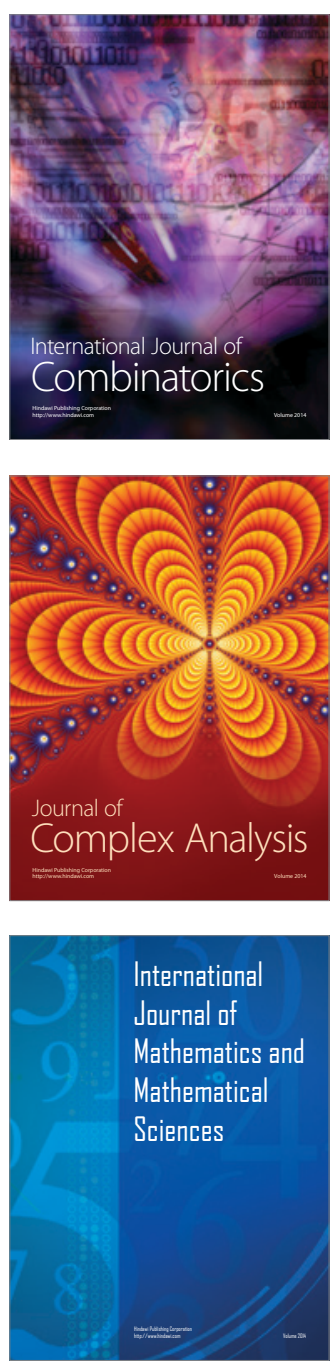
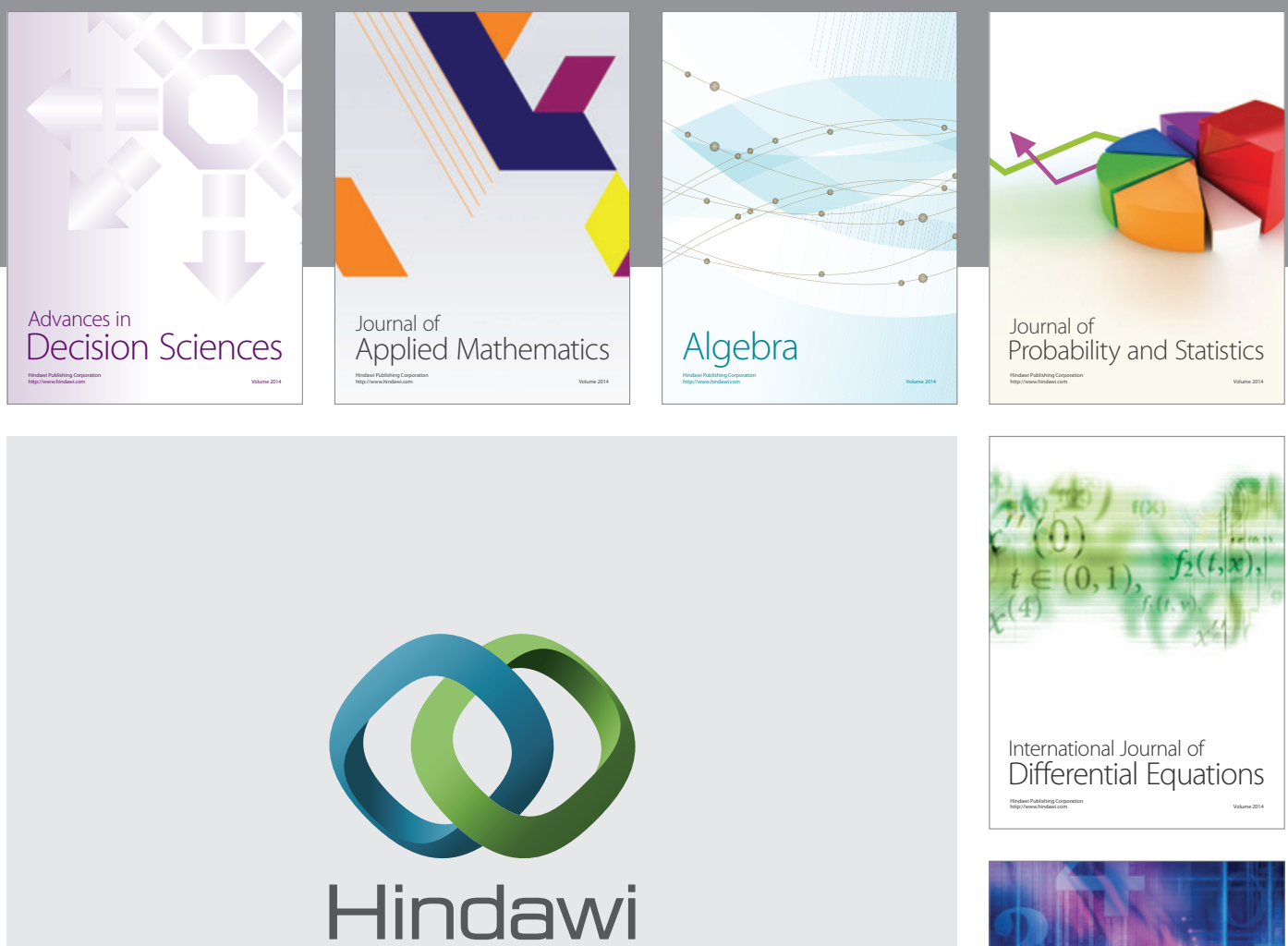

Submit your manuscripts at http://www.hindawi.com
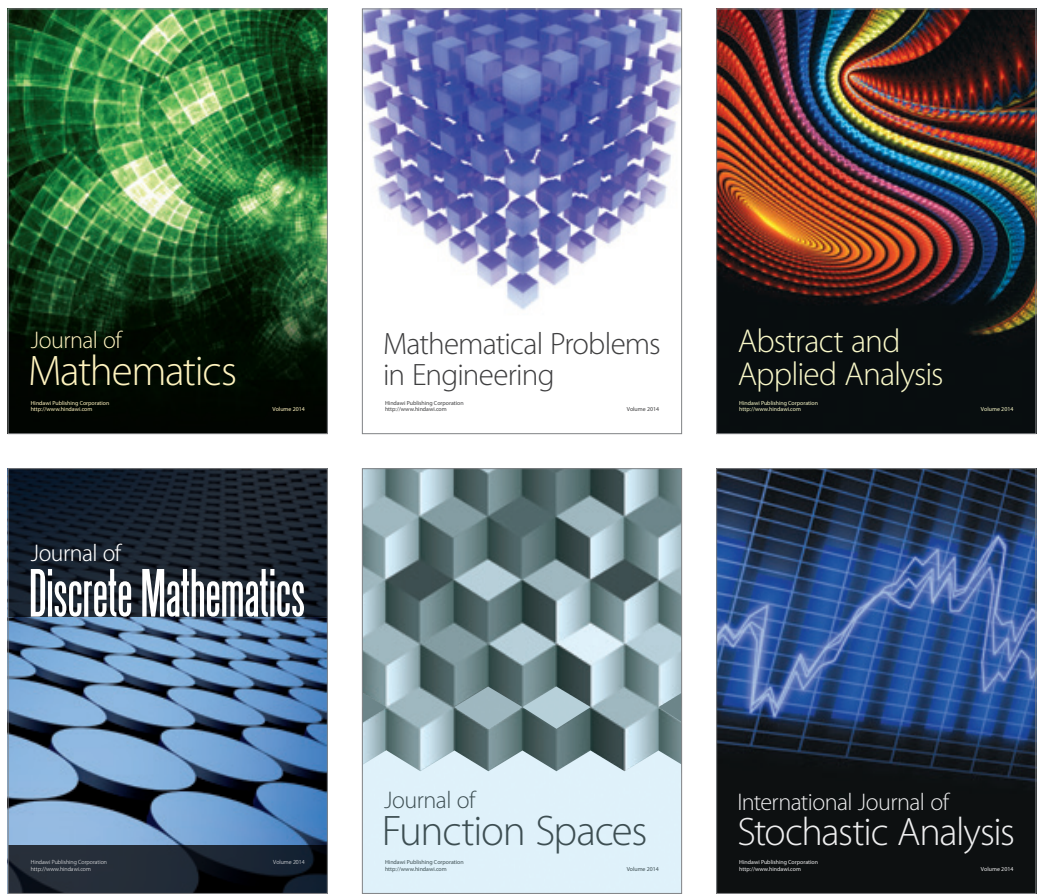

Journal of

Function Spaces

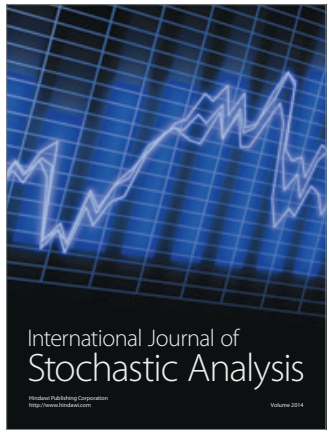

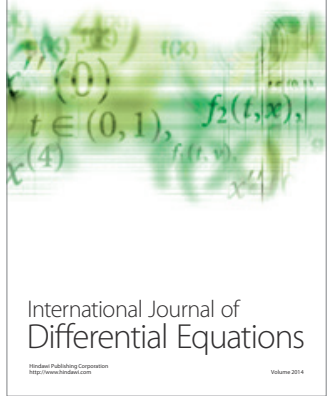
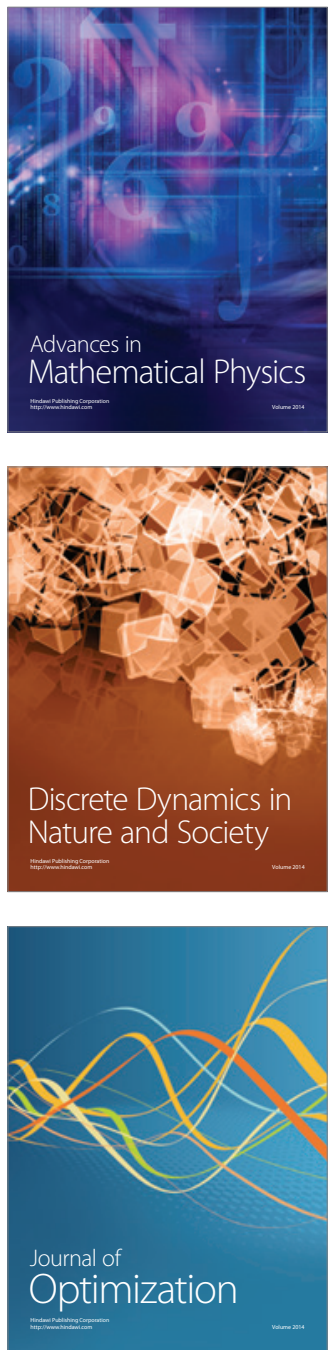